\title{
Omental Adipose Removal Decreases High Blood Pressure in Hypertensive Patients Independent of Body Mass Index
}

\author{
Li Jiang ${ }^{1, *}$ \\ Wei Sun ${ }^{2, *}$ \\ Mi Zhang ${ }^{3, *}$ \\ Yaqing Wang ${ }^{2}$ \\ Yunfan $\operatorname{Tian}^{2}$ \\ Peng $\mathrm{Li}^{2}$ \\ Yan Lu ${ }^{2}$ \\ Tianhua $X u^{2}$ \\ Ming Qiu ${ }^{2}$ \\ Yun Yang ${ }^{2}$ \\ Xuemei Jia ${ }^{3}$ \\ Xiangqing Kong ${ }^{2}$ \\ 'Department of Pediatrics, The First \\ Affiliated Hospital of Nanjing Medical \\ University, Nanjing, Jiangsu, People's \\ Republic of China; ${ }^{2}$ Department of \\ Cardiology, The First Affiliated Hospital of \\ Nanjing Medical University, Nanjing, Jiangsu, \\ People's Republic of China; ${ }^{3}$ Department of \\ Gynecological Tumor, Nanjing Maternity and \\ Child Health Hospital, Nanjing, Jiangsu, \\ People's Republic of China \\ *These authors contributed equally to this \\ work
}

Correspondence: Xiangqing Kong

The First Affiliated Hospital of Nanjing

Medical University, 300 Guang Zhou

Road, Nanjing, Jiangsu Province, 210029,

People's Republic of China

Tel +862583718836

Fax +862583724440

Email kongxq@njmu.edu.cn

Xuemei Jia

The Affiliated Obstetrics and Gynecology Hospital of Nanjing Medical University (Nanjing Maternity and Child Health

Hospital), 123 Tianfeixiang, Mochou

Road, Nanjing, Jiangsu Province, 210004 ,

People's Republic of China

Tel/Fax +86 2584460507

Emailxmjia@njmu.edu.cn
Aim: Several studies have demonstrated that increased omental adipose is a risk factor for obesity and metabolic syndrome. It remains unclear whether it is responsible for hypertension as an independent risk. This study was designed to assess the impact of omental adipose removal by surgery on blood pressure in cancer patients with or without hypertension.

Methods and Results: In this prospective observational study, 133 patients with gastric or gynecological cancer were divided into 3 groups: non-hypertensive and omentum removed (NH\&OR), hypertensive and omentum removed (H\&OR), and hypertensive and omentum present (H\&OP). Patients were followed up with systolic and diastolic blood pressure (SBP and DBP), changes in related body mass index and metabolic indices. The time points of the 2 follow-up visits were 1 month \pm 7 days after the operation before the start of chemotherapy and the endpoint of $8 \pm 1$ month. Omental adipose tissues from both non-hypertensive and hypertensive patients in surgery were collected. We included 133 patients $(84.2 \%$ female, $20.3 \%$ malignant gastric cancer and $79.7 \%$ malignant gynecological cancer, $78.2 \%$ omentum removal, $48.9 \%$ hypertensive), and all completed follow-up. H\&OR group showed significant reductions in systolic and diastolic blood pressure compared with the baseline at $1-\mathrm{m}(-16.94 /-10.50 \mathrm{mmHg}$, both $P<0.001)$ and $8-\mathrm{m}$ end point $(-16.00 /-5.50 \mathrm{mmHg}, P<0.001$ and $P=0.004)$. Little reductions were observed with the body mass index of patients in 3 groups till the endpoint of study (H\&OR group: $24.60 \mathrm{~kg} / \mathrm{m}^{2}$ to $23.57 \mathrm{~kg} / \mathrm{m}^{2}$, NH\&OR group: $23.45 \mathrm{~kg} / \mathrm{m}^{2}$ to $23.25 \mathrm{~kg} / \mathrm{m}^{2}$, H\&OP group: $25.74 \mathrm{~kg} / \mathrm{m}^{2}$ to $25.24 \mathrm{~kg} /$ $\mathrm{m}^{2}$, all $P>0.05$ ). No correlation was found between the baseline body mass index and 8-m change of systolic and diastolic blood pressure in omentum removed groups. In both groups, triglyceride levels were significantly increased at $4 \pm 1$ week after surgery (NH\&OR $0.32 \mathrm{mmol} / \mathrm{L}, P=0.006$; H\&OR $0.40 \mathrm{mmol} / \mathrm{L}, P=0.010$ ).

Conclusion: Resection of omental adipose tissue represents an effective strategy for reducing systolic and diastolic blood pressure at 8 months in hypertensive patients, even in the non-obese hypertensive population.

Keywords: hypertension, omentum adipose, mRNA microarray

\section{Introduction}

Hypertension is a worldwide epidemic. More than $50 \%$ of the population older than 60 years old has hypertension in many countries, and this prevalence is still steadily increasing with the use of antihypertensive medications. ${ }^{1}$ The estimated number of years of deaths caused by hypertension-related diseases, including ischemic heart disease, stroke, chronic kidney disease, and other circulatory diseases, was up to 11.3 million combined in $2010 .^{2,3}$ More than $65 \%$ of patients with hypertension are overweight or show a tendency for obesity in the incidence of obesity hypertension. 
Obesity is a major risk factor for many cardiovascular diseases. Obesity has direct and indirect effects on worsening arterial hypertension, atherosclerosis, and related metabolic syndrome, including insulin resistance and type 2 diabetes. $^{4-6}$

Recent studies have shown that the intra-abdominal adipose tissue depot, which is mainly made up of visceral intraperitoneal fat, primarily omental and mesenteric fat, is strongly associated with cardiovascular risk factors. ${ }^{7,8}$ Studies on mice and randomized, controlled studies in humans have shown that surgical visceral fat (VF) removal or reducing the body mass index (BMI) by an operation can treat metabolic syndrome and control hypertension in patients with obesity. ${ }^{9,10}$ However, there is still uncertainty regarding the relative importance of VF, especially omental adipose tissue, for the hypertensive population.

Therefore, we designed this prospective observational study to assess the effect of surgery with omental adipose tissue removal on hypertensive patients with stomach cancer or ovarian cancer.

\section{Materials and Methods}

We performed a multicenter, prospective observational study. We included 2 clinical centers of Jiangsu Province Hospital and Nanjing Maternity and Child Health Hospital, which are both located in Jiangsu Province, China.

\section{Study Participants}

Patients aged 20-80 years $\left(\mathrm{BMI} \geq 18\right.$ and $<40 \mathrm{~kg} / \mathrm{m}^{2}$ ) with or without a documented history of hypertension were included. Patients with gastric cancer were from the
General Surgery Department at Jiangsu Province Hospital. Patients with gynecological, including ovarian, cervical and endometrial, cancer were from the Gynecology Department at Nanjing Maternity and Child Health Hospital. The study population included consecutive patients who presented for a tumor resection operation in which greater omental tissue was completely removed.

Patients who met the following criteria were included in the hypertensive group: systolic blood pressure (SBP) $\geq 140 \mathrm{mmHg}$ and/or diastolic blood pressure (DBP) $\geq 90$ mmHg, ${ }^{11}$ and exclusion of secondary hypertension. Exclusion criteria included the following: (1) women in lactation or pregnancy; (2) patients with echocardiographic abnormalities, signs of severe arrhythmia, or a prolonged period of correction of QT; (3) patients with severe cardiovascular disease occurring within 6 months of study entry, including uncontrolled congestive heart failure, unstable angina pectoris, or acute myocardial infarction; (4) patients with abnormal coagulation function; and (5) patients unwilling to participate in this study.

\section{Study Design and Endpoints}

The study design is shown in Figure 1. The study duration was 18 months of treatment and follow-up, from February 2016 to August 2017. All eligible patients formed 3 groups: non-hypertensive and omentum removed (NH\&OR), hypertensive and omentum removed (H\&OR), and hypertensive and omentum present (H\&OP). All the patients were followed up for changes in office blood pressure, and changes in related clinical and metabolic indices. The time points of the 2 follow-up visits were 1

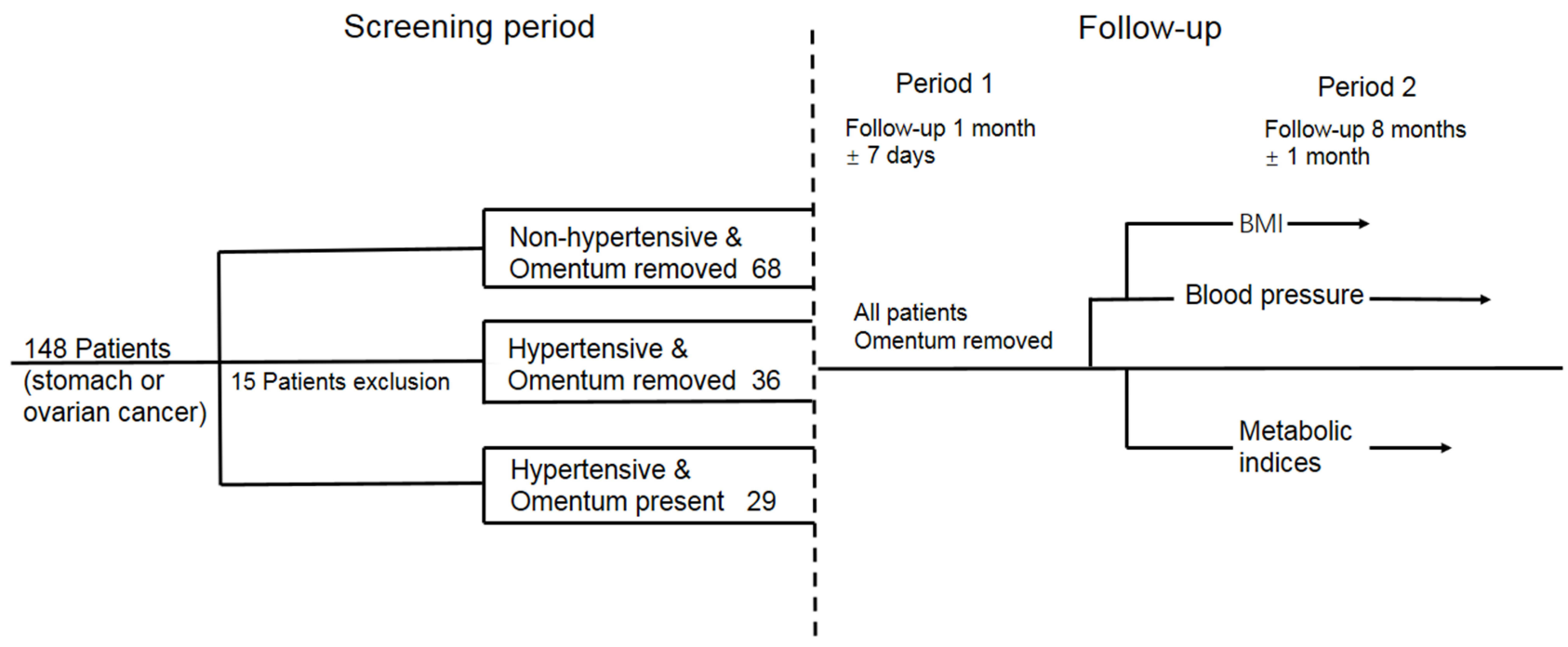

Figure I Study design flow. 
month \pm 7 days (1-m) after the operation before the start of chemotherapy and $8 \pm 1$ month $(8-\mathrm{m})$ after the operation at least 2 months after the end of chemotherapy. Antihypertensive drugs were used until the day of surgery. The patients' blood pressure would be reassessed after the surgery, which was for deciding whether or not to give anti-hypertension medication and give intravenously or orally.

The main endpoints were accomplished by outpatient follow-up or telephone visits with the patients or their first family members 2 months after their last chemotherapy ended. The main secondary outcomes for the core study included analysis of correlation between changes in blood pressure (BP) and BMI. Changes in the metabolic index of blood chemistry were recoded.

\section{Assessments and Clinical Outcome Measurements}

The primary outcome was a change in mean office BP from baseline to 1 and 8 months to evaluate the effects of surgical removal of omental adipose on BP. Arterial BP measurements were obtained using a calibrated standard mercury sphygmomanometer or an aneroid sphygmomanometer with an appropriately sized cuff. SBP was recorded when the initial heart sound was heard (Phase I of the Korotkoff sound) and DBP was recorded at the disappearance of the heart sound (Phase V of the Korotkoff sound). BP was measured in the same arm at each evaluation, preferably in the right arm. During each follow-up, office $\mathrm{BP}$ was measured 3 times at 1-min to 2-min intervals, and the final average data were recorded.

The change in BMI was assessed from baseline to the endpoint of the study as a major referenced objective related to the change in BP. Regular monitoring of blood chemistry, including serum albumin, total bilirubin, direct bilirubin, total cholesterol, triglyceride, fasting blood glucose, and uric acid levels, was performed before the operation and after the acute trauma period of post-surgery (mean of $4 \pm 1$ weeks postoperatively before chemotherapy). Patients visited the 2 centers as a regular visit every 3 weeks after surgery for regular chemotherapy and physical examinations.

\section{Ethics Review}

This study followed the principles outlined in the Helsinki declaration and was conducted according to Good Clinical Practice standards established by the International
Conference on Harmonization. The study protocol and informed consent were reviewed by the institutional review board or ethics committee at each site. Informed consent and, where applicable, assets were obtained from all participants and their parents or legal guardians according to local standards. Safety assessments included monitoring and recording all adverse events and serious adverse events. Regular blood chemistry and physical examinations were performed. Additional information on adverse events was collected if volunteered at other times during the study.

\section{Statistical Methods}

SPSS22.0 (SPSS Inc., Chicago, IL, USA) for Windows was used to analyze all the recorded data. Descriptive data that follow a skewed distribution are presented as numbers with percentage and median with range. Data with a normal distribution are shown as mean $\pm \mathrm{SD}$, as appropriate, and frequencies are used to describe categorical variables. Differences in data between the groups that followed a normal distribution were tested with ANOVA and the SNK test for multiple comparisons. The Wilcoxon rank-sum test was used for variables with a skewed distribution, and the chi-square test was used for categorical variables. Differences between baseline and follow-up assessments were tested with paired $t$-tests. The Pearson test was used to analyze the correlation between BMI and the change in BP. A 2-tailed $P<0.05$ was used as a criterion for statistical significance. The difference value is expressed as the postoperative value minus the preoperative value.

\section{Results \\ Core Study}

\section{Baseline Characteristics}

Of 148 eligible participants, 133 (89.9\%) were included. Baseline measurements, metabolic indices, and medications are shown in Table 1. Baseline characteristics were similar between the 3 groups regarding age, the sex ratio, BMI, and heart rate. Office SBP and DBP were not significantly different between the two hypertensive groups. The four metabolic indices of total bilirubin, total cholesterol, triglyceride, and fasting blood-glucose levels were significantly lower in the NH\&OR group compared to the other 2 hypertensive groups $(P=0.039 ; P=0.042 ; P=0.010 ; P<0.001)$. There were 3 and 2 patients in each hypertensive group who 
Table I Baseline Characteristics of Studied Subjects

\begin{tabular}{|c|c|c|c|c|}
\hline Variable & NH \& OR $(n=68)$ & H \& OR $(n=36)$ & H \& OP $(n=29)$ & $P$ value \\
\hline Age, y & $58.4 \pm 7.6$ & $61.0 \pm 8.6$ & $60.5 \pm 7.1$ & 0.22 \\
\hline Male sex, n (\%) & $13(19)$ & $8(23)$ & $0(0)$ & 0.028 \\
\hline $\mathrm{BMI}, \mathrm{kg} / \mathrm{m} 2$ & $23.45 \pm 0.64$ & $24.60 \pm 0.69$ & $25.74 \pm 0.52$ & 0.170 \\
\hline Heart rate, bpm & $65 \pm 11$ & $68 \pm 12$ & $67 \pm 11$ & 0.53 \\
\hline Office SBP, mmHg & $116.69 \pm 11.13$ & $|39.89 \pm| \mid .82$ & $136.50 \pm 8.30 *$ & $<0.01$ \\
\hline Office DBP, $\mathrm{mmHg}$ & $75.22 \pm 7.83^{\#}$ & $86.69 \pm 9.04$ & $83.77 \pm 6.69$ & $<0.01$ \\
\hline Serum albumin, $g / l$ & $40.40(38.35-42.15)$ & $41.3(39.5-43.6)$ & $42.0(39.7-44.7)$ & 0.134 \\
\hline Total bilirubin, $\mu \mathrm{mol} / \mathrm{L}$ & $8.90(7.10-11.70)$ & $10.8(8.6-12.9)$ & $9.55(8.40-13.80)$ & 0.039 \\
\hline Direct bilirubin, $\mu \mathrm{mol} / \mathrm{L}$ & $2.9(2.3-4.0)$ & $3.3(2.7-4.4)$ & $3.4(2.9-4.1)$ & 0.092 \\
\hline Total cholesterol, $\mathrm{mmol} / \mathrm{L}$ & $4.4 I(3.73-5.03)$ & $4.73(4.23-5.27)$ & $4.87(4.21-5.30)$ & 0.042 \\
\hline Triglyceride, $\mathrm{mmol} / \mathrm{L}$ & I.II(0.73-I.49) & $1.39(1.12-1.83)$ & $1.37(0.96-2.09)$ & 0.010 \\
\hline Fasting blood-glucose, $\mathrm{mmol} / \mathrm{L}$ & $4.83(4.44-5.10)^{\#}$ & $5.21(5.00-6.00)$ & $5.36(4.89-6.40)$ & $<0.001$ \\
\hline Uric Acid, $\mu \mathrm{mol} / \mathrm{L}$ & $236.0(194.2-281.6)$ & $239.8(210.1-292.9)$ & $290.1(205.3-328.8)$ & 0.064 \\
\hline Antihypertensive Drugs & & & & $>0.05$ \\
\hline Beta-blockers, n (\%) & - & $18(58)$ & $18(69)$ & 0.331 \\
\hline ACE-I/ARB, n (\%) & - & $25(80)$ & $19(73)$ & 0.736 \\
\hline Diuretics, n (\%) & - & $10(32)$ & $9(35)$ & 0.146 \\
\hline Calcium-channel blockers, n (\%) & - & $13(42)$ & $13(50)$ & 0.476 \\
\hline Alpha-I blockers, n (\%) & - & $5(16)$ & $7(27)$ & 0.290 \\
\hline Untreated agents, $n(\%)$ & & $5(13)$ & $3(10)$ & 0.958 \\
\hline
\end{tabular}

Notes: Values are shown as means \pm SD or medians (interquartile range) or absolute numbers and percentages. ACE-I indicates angiotensin converting enzyme. ARB indicates angiotensin receptor blocker. ${ }^{*}$ Compared with $\mathrm{NH} \&$ OR group and $\mathrm{H} \&$ OR group. ${ }^{\#}$ Compared with $\mathrm{H} \& \mathrm{OR}$ and $\mathrm{H} \&$ OP group.

did not accept any antihypertensive therapy. Table 2 shows the distribution of clinical diagnosis in each group.

\section{Change in BP}

Changes in office SBP and DBP from baseline to 1 month and 8 months in all the patients were analyzed (Table 3 and Figure 2). A significant reduction in office SBP and DBP was found at 1 month $(-16.94 /-10.50 \mathrm{mmHg})$ and 8 months $(-16.00 /-5.50 \mathrm{mmHg})$ compared with baseline in the H\&OR group (all $P<0.05$ ). However, in the NH\&OR group, there was only a slight nonsignificant reduction in office SBP and DBP at 1 and 8 months. In the H\&OP group, there was also no significant decrease in BP. Furthermore, in the H\&OP group, office DBP was even slightly increased compared with baseline at the end of 8 months. These data were all normally distributed.

\section{BMI Correlations}

BMI of each patient was calculated to assess the effects of body weight on BP and the relationship between BMI and changes in BP in patients with omentum removal. Compared with baseline, a slight nonsignificant reduction in BMI of patients in the 3 groups was observed until the endpoint ( 8 months) of the study (H\&OR group: $24.60 \mathrm{~kg} / \mathrm{m}^{2}$ to $23.57 \mathrm{~kg} / \mathrm{m}^{2}$, NH\&OR group: $23.45 \mathrm{~kg} /$ $\mathrm{m}^{2}$ to $23.25 \mathrm{~kg} / \mathrm{m}^{2}$, H\&OP group: $25.74 \mathrm{~kg} / \mathrm{m}^{2}$ to $25.24 \mathrm{~kg} / \mathrm{m}^{2}$, all $P>0.05$ ) (Figure $3 \mathrm{~A}$ ). Figure $3 \mathrm{~B}$ and $\mathrm{C}$ shows the correlations between baseline BMI and the change in SBP and DBP at 8 months in the H\&OR and NH\&OR groups. We found that there was no significant correlation between baseline BMI and a change in BP at 8 months $(\mathrm{r}=-0.099, P=0.322$ for SBP; $\mathrm{r}=-0.004$, $P=0.663$ for DBP).

Table 2 Distribution of Disease in $\mathrm{NH} \& \mathrm{OR}, \mathrm{H}$ \& OR and $\mathrm{H}$ \& OP Groups

\begin{tabular}{|l|l|l|l|}
\hline Variable & NH \& OR (n=68) & H \& OR (n=36) & H \& OP (n=29) \\
\hline Malignant gastric tumor, n (\%) & $17(25)$ & $10(28)$ & - \\
Malignant ovarian tumor, n (\%) & $51(75)$ & $26(72)$ & - \\
Cervical cancer, n (\%) & - & - & $15(52)$ \\
Endometrial cancer, n (\%) & - & - & $14(48)$ \\
\hline
\end{tabular}


Table 3 Changes in Office SBP and Office DBP from Baseline to End of Period I and Period 2 in 3 Groups

\begin{tabular}{|l|l|l|l|l|}
\hline Variable & $\begin{array}{l}\text { Non-Hypertensive and } \\
\text { Omentum Removed }(\mathbf{n}=\mathbf{6 8})\end{array}$ & $\begin{array}{l}\text { Hypertensive and Omentum } \\
\text { Removed }(\mathbf{n = 3 6})\end{array}$ & $\begin{array}{l}\text { Hypertensive and } \\
\text { Omentum Kept }(\mathbf{n}=\mathbf{2 9})\end{array}$ & $\boldsymbol{P}$ value \\
\hline Diff SBP in Im & $-1.00(-13.50 \sim 5.00)$ & $-16.94(-24.50 \sim-8.00)^{\mathrm{ab}}$ & $-1.00(-6.00 \sim 3.00)$ & $<0.00 \mathrm{I}$ \\
Diff SBP in 8m & $-2.74(-12.00 \sim 7.00)$ & $-16.00(-24.00 \sim-5.00)^{\mathrm{ab}}$ & $-4.00(-8.00 \sim 5.00)$ & $<0.00 \mathrm{I}$ \\
Diff DBP in Im & $-1.00(-10.00 \sim 5.00)$ & $-10.50(-14.50 \sim-4.50)^{\mathrm{ab}}$ & $-2.00(-7.00 \sim 6.00)$ & $<0.00 \mathrm{I}$ \\
Diff DBP in $8 \mathrm{~m}$ & $-1.00(-6.00 \sim 6.00)$ & $-5.50(-11.00 \sim 0.00)^{\mathrm{ab}}$ & $0.50(-4.00 \sim 6.00)$ & 0.004 \\
\hline
\end{tabular}

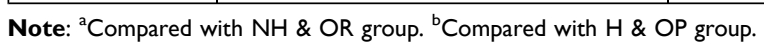

\section{Changes in Metabolic Indices}

Table 4 shows the difference value of metabolic indices changes, before and after the operation $(4 \pm 1$ weeks postoperatively before chemotherapy) in each group. Comparison between groups: Two indices, difference value of serum albumin and total bilirubin, showed significant variation when 3 groups compared simultaneously $(P=$ 0.019; $P=0.025$ ). However, there was no significant difference in serum albumin (NH\&OR vs $\mathrm{H} \& \mathrm{OR}, P=1.000$; NH\&OR vs H\&OP, $P=0.052 ; \mathrm{H} \& \mathrm{OR}$ vs $\mathrm{H} \& \mathrm{OP}, P=0.149$ ) or in total bilirubin (NH\&OR vs H\&OR, $P=0.108$;
NH\&OR vs H\&OP, $P=0.061$; H\&OR vs H\&OP, $P=$ $1.000)$ between each of the 2 groups.

Comparison within each group pre-/post-operatively: In the H\&OR group, triglyceride levels were significantly increased $(P=0.010)$, and serum albumin, total bilirubin, and direct bilirubin levels were decreased to varying degrees postoperatively compared with preoperatively $(P=0.001 ; P<$ $0.001 ; P=0.006)$. In the NH\&OR group, triglyceride levels were also significantly increased $(P=0.006)$, and only serum albumin levels were significantly decreased postoperatively compared with preoperatively $(P=0.004)$. In the H\&OP

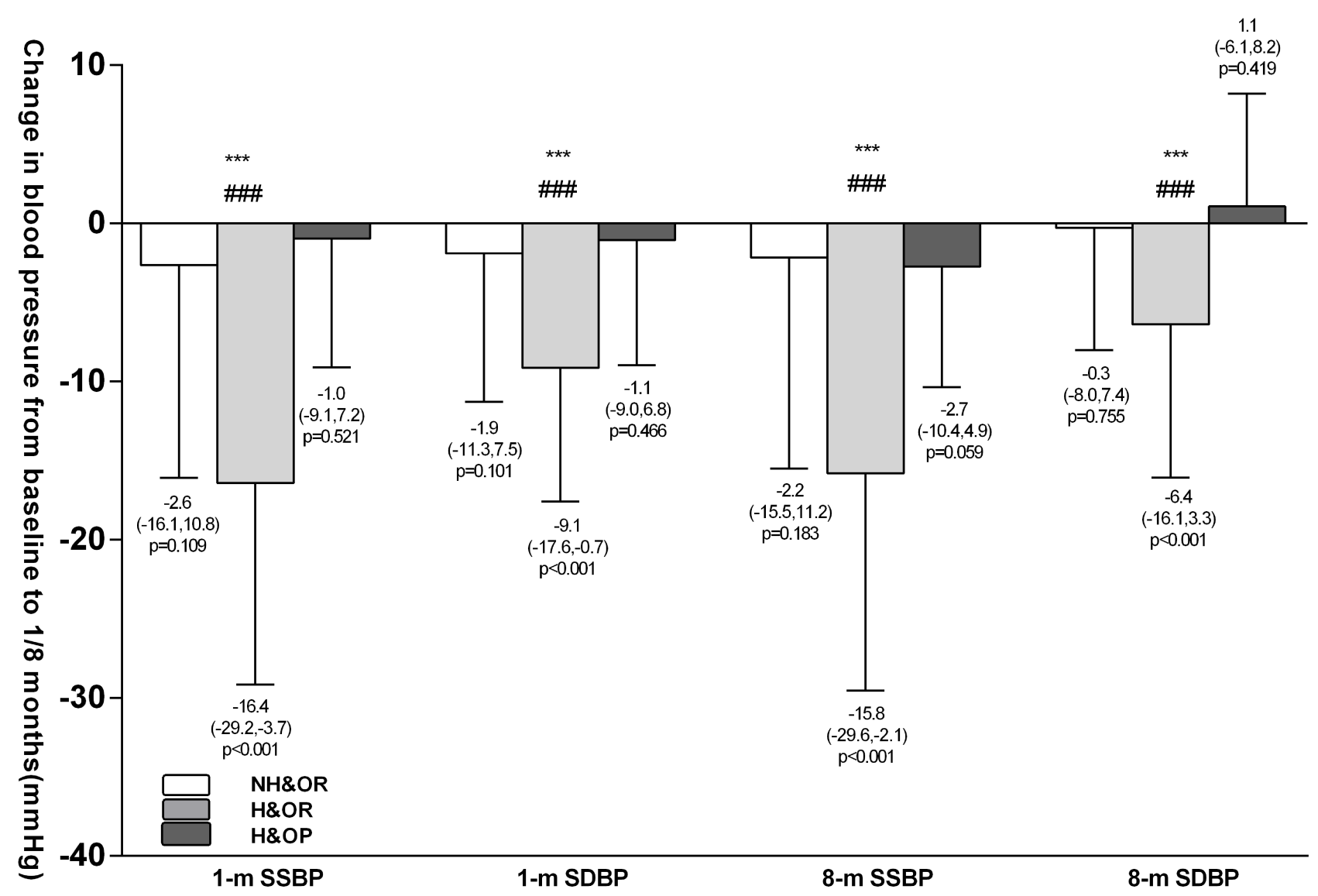

Figure 2 Changes of office SBP and DBP from baseline to the $1-\mathrm{m}$ and 8-m end point in 3 groups. (Between group differences and blood pressure differences from baseline to the I month and 8-month follow-up assessment were tested using unpaired and paired $t$ tests, respectively.) $(* * * p<0.00$ I H\&OR group vs NH\&OR group, \#\# $p<0.00 \mathrm{I}$ H\&OR group vs H\&OP group). 
A

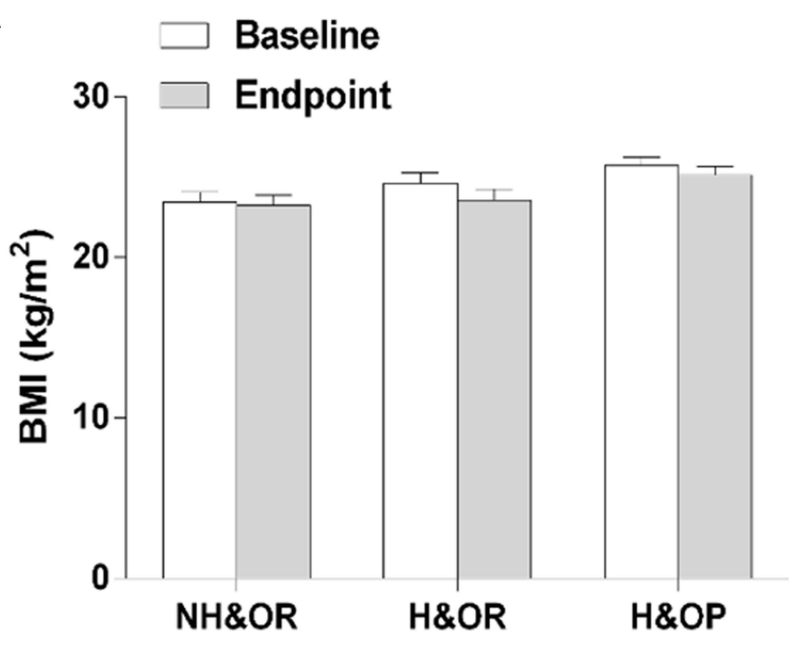

B

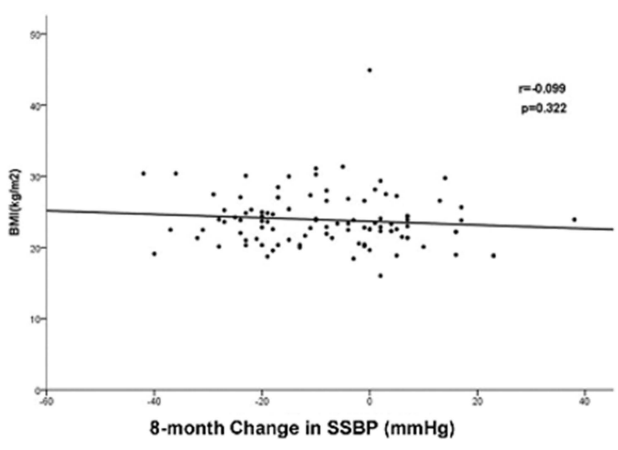

C

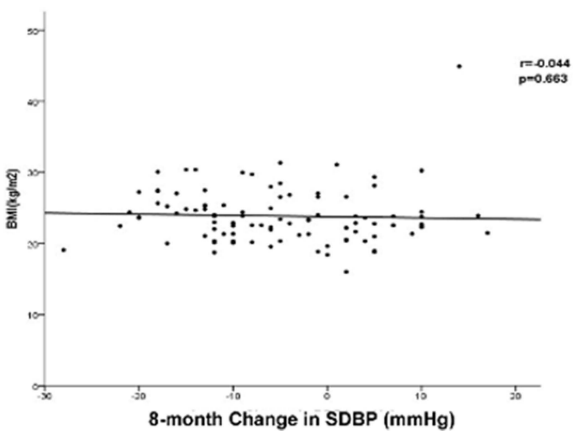

Figure 3 (A) Change of BMI in 3 group at the endpoint of 8-m. (B) Correlation between the baseline of BMI and the change of SBP in H\&OR and NH\&OR groups. (C) Correlation between the baseline of BMI and the change of DBP in H\&OR and NH\&OR groups.

group, 4 indices were significantly improved after surgery, including serum albumin, total bilirubin, direct bilirubin and fasting blood glucose levels $(P<0.001 ; P=0.001 ; P=0.006$; $P=0.046)$. Compared with the H\&OR group, the downward trend of the first 3 metabolic indicators in the H\&OP group was similar, while triglyceride levels did not significantly change. In the H\&OP group, postoperative fasting blood glucose level decreased significantly compared to preoperative period $(P=0.046)$.

Among the 3 groups, metabolic markers in the NH\&OR group showed the least significant changes before and after the operation.

\section{Discussion}

Our study showed that at 8 months, patients with stomach or ovarian cancer with hypertension who underwent radical surgery with total removal of the greater omentum exhibited a significant reduction of $16.0 \mathrm{mmHg}$ in SBP and $5.5 \mathrm{mmHg}$ in DBP. Non-hypertensive patients with omental removal and hypertensive patients without omental removal experienced remarkable changes after surgery. Notably, 3 patients in the H\&OR group were able to maintain stable normal blood pressure without relying on oral antihypertensive drugs (remission of hypertension), whereas no patients in the H\&OP group were free of

Table 4 Changes of Metabolic Indexes in NH \& OR, H \& OR and H \& OP Groups

\begin{tabular}{|c|c|c|c|c|}
\hline Variable & $\begin{array}{l}\text { Hypertensive and } \\
\text { Omentum Removed } \\
(n=68)\end{array}$ & $\begin{array}{l}\text { Non-Hypertensive and } \\
\text { Omentum Removed }(n=36)\end{array}$ & $\begin{array}{l}\text { Hypertensive and } \\
\text { Omentum Kept } \\
(n=29)\end{array}$ & $P$ value \\
\hline Diff in Serum albumin $(g / l)$ & $-3.10(-7.8 \sim-0.3)^{\#}$ & $-2.55(-7.30 \sim 1.00)^{\#}$ & $-7.10(-10.45 \sim-3.70)^{\#}$ & 0.019 \\
\hline Diff in Total bilirubin $(\mu \mathrm{mol} / \mathrm{L})$ & $-3.10(-4.95 \sim-1.05)^{\#}$ & $-0.80(-4.15 \sim 1.55)$ & $-2.8(-7.0 \sim 0.1)^{\#}$ & 0.025 \\
\hline Diff in Direct bilirubin $(\mu \mathrm{mol} / \mathrm{L})$ & $-0.95(-1.75 \sim 0.15)^{\#}$ & $-0.40(-1.15 \sim 0.75)$ & $-0.5(-2.0 \sim 0.3)^{\#}$ & 0.237 \\
\hline Diff in Total cholesterol (mmol/L) & $-0.17(-1.06 \sim 0.53)$ & $0.11(-0.74 \sim 0.53)$ & $-0.32(-0.89 \sim 0.13)$ & 0.334 \\
\hline Diff in Triglyceride ( $\mathrm{mmol} / \mathrm{L})$ & $0.40(-0.11 \sim 0.75)^{\#}$ & $0.32(0.03 \sim 0.70)^{\#}$ & $0.28(-0.11 \sim 0.60)$ & 0.700 \\
\hline Diff in Fasting blood-glucose $(\mathrm{mmol} / \mathrm{L})$ & $0.01(-0.81 \sim 0.46)$ & $0.19(-0.20 \sim 0.55)$ & $-0.30(-0.80 \sim 0.50)^{\#}$ & 0.247 \\
\hline Diff in Uric Acid ( $\mu \mathrm{mol} / \mathrm{L})$ & $8.8(-66.5 \sim 56.8)$ & $-19.85(-87.80 \sim-6.40)$ & $-3.4(-28.1 \sim 77.7)$ & 0.326 \\
\hline
\end{tabular}

Note: "The difference between post- and preoperative was statistically significant. 
antihypertensive medications at 8 months. Although patients in our study underwent surgery and chemotherapy, they did not experience a significant change in BMI at the endpoint of the study. This finding indicated that the decline in SBP and DBP in the H\&OR group was not caused by a substantial weight loss. This change in BP did not occur in patients with normal BP and it did not occur in patients who underwent the same operation but did not have a resection of omental adipose. Additionally, in patients in the H\&OR and NH\&OR groups, there was no significant correlation between a decrease in BP and the original BMI at the study endpoint.

In both omental adipose removal groups, an increase in triglyceride levels was observed, while patients without omental adipose tissue removal did not experience this change. All 3 groups of patients showed an obvious decrease in serum albumin levels after surgery compared with before surgery. Interestingly, patients who underwent omental adipose removal during surgery showed an improvement in triglyceride levels. However, resection of omental adipose did not affect fasting blood glucose levels in patients with omentum removal. In patients without omental adipose resection, there was a significant decrease in fasting blood glucose levels, but no significant change in triglyceride levels postoperatively. Comparison of these changes showed that removal of omental adipose could affect lipid metabolism in the body. Compared with nonhypertensive patients, hypertensive patients who underwent omental adipose resection were more likely to experience changes in certain metabolic indicators.

Several observational studies have suggested a relationship on improvement and remission between hypertension and visceral adipose. ${ }^{12}$ Abdominal obesity is more closely associated with the risk of disease than excess total body fatness per se. ${ }^{13,14}$ As an independent organ, the omentum is a visceral adipose tissue with unique immune and inflammation function, which is closely related to metabolism of the organism.

In humans, the omentum is large, reaching an area of $1500 \mathrm{~cm}^{2}{ }^{15}$ The omentum contains not only a large amount of white fat but also many blood vessels and sympathetic fibers. Although the effect of the omentum is poorly understood and usually overlooked, the omentum is immunologically important. Conroy et al suggested that the omentum contributes to peritoneal immunity by collecting particulates and antigens to promote a variety of immune responses, including inflammation or fibrosis. ${ }^{16}$ Reciprocal interactions between lymphoid aggregates and adipocytes in the omentum regulate systemic inflammation and metabolic function. Importantly, this immune function may occur in the context of obesity. Moreover, omental adipose tissue is enriched with inflammatory macrophages and $\mathrm{T}$ cells mediating pathological inflammation. Expression of chemokines and receptors in proinflammatory $\mathrm{T}$ cells are implicated in inflammatory diseases, such as atherosclerosis, hypertension, and asthma. ${ }^{16-18}$ Recent research showed that a characteristic sympatho-excitatory reflex was present in white adipose tissue named the adipose afferent reflex, which contributed to hypertension only when sympathetic nerves in white adipose tissue were abnormally activated. ${ }^{19,20}$ Blockade of an enhanced adipose afferent reflex in patients with obesity and hypertension helps to reduce sympathetic outflow and attenuates hypertension.

Our study showed that resection of omental adipose tissue only had the effect of reducing BP in hypertensive patients but not in normotensive patients. This phenomenon indicates that omental adipose tissue may be the target of an unusually high BP. However, in normotensive patients, the role of this target is not activated. Furthermore, in our study, there was no significant correlation between this effect on BP and BMI in hypertensive patients. Moreover, measurements of epinephrine, norepinephrine, and dopamine levels in omental adipose tissue showed that the decline in BP was not caused by a decrease in local catecholamine levels.

A considerable number of studies have reported that the incidence and development of hypertension are positively correlated with BMI. A previous study by Chandra et al showed that BP in patients with obesity and hypertension who had a significant reduction in BMI by undergoing Roux-en-Y gastric bypass plus medical therapy could be better controlled compared with patients who had only antihypertensive medication. ${ }^{7}$ Our study showed different results from this previous study. The current study showed that a decrease in BP was not associated with the original BMI and changes in BMI during followup. Omental adipose tissue is a specific risk factor for hypertensive patients independent of BMI or even obesity. In our study, there was no association between BMI and omental adipose tissue. The study by Chandra et al showed that when VAT, subcutaneous adipose tissue (SAT), and lower body fat (LBF) were added to the model of severely obese patients undergoing bariatric surgery, only VAT remained independently, but not total or subcutaneous adiposity, which was associated with incident 
hypertension. The strongest associations were observed with retroperitoneal adipose tissue. ${ }^{7}$

An increasing number of studies are supporting the notion that omental adipose tissue is an independent cardiovascular risk factor by affecting inflammation and metabolism of the body. Hardy et al showed that increased infiltration of macrophages and adipocytes of the omentum is associated with insulin resistance but not simply BMI in patients with obesity. ${ }^{21}$ A study by Rosenquist et al suggested that a decrease in attenuation or increase in volume of abdominal adipose is an independent risk factor for hypertension, hypertriglyceridemia, and metabolic syndrome. ${ }^{22}$ Another study showed that the intraabdominal adipose area remained a significant risk factor for hypertension in Japanese Americans and this was not reflected by fasting plasma insulin levels. ${ }^{23}$ This finding is consistent with our research. In both groups of patients with omental removal, there were no significant changes in fasting blood glucose levels. The mechanism by which omental adipose tissue is associated with hypertension remains to be determined. de Lemos et al pointed out that VAT may be the primary driver of metabolic and cardiovascular complications. ${ }^{23}$ We should focus on more targeted interventions to prevent a gain in VAT or support VAT loss but not generalized weight loss.

Theoretically, lipid storage is a risk factor for the development of metabolic complications and cardiovascular dysfunction. In women with obesity, the amount of VAT is associated with circulating triglyceride concentrations. ${ }^{24}$ However, in our study, circulating triglyceride levels were dramatically elevated after omental resection. Foster et al showed that, in rodents, VF removal could significantly decrease hepatic lipoprotein lipase and triglyceride levels, and improve glucose tolerance. ${ }^{25}$ However, their follow-up period was only 8 weeks. We speculate that removing omental adipose tissue for a short period of time causes compensatory activation of liver fat metabolism, consequently promoting synthesis of triglycerides. This could then lead to a short-term elevation of postoperative triglyceride levels in the circulation. Due to limitations of chemotherapy, the long-term changes in triglyceride levels cannot be fully associated with resection of the omentum. Therefore, the long-term changes in blood lipid and blood glucose levels still need to be verified.

Several limitations of our study must be acknowledged. First, we could only perform observational studies in certain patients with tumors, and the follow-up was only 8 months. We attempted to minimize the potential errors caused by surgery and chemotherapy by including a control group. Second, due to the objective conditions, ambulatory blood pressure monitoring (ABPM) was not performed on every participant in our trial. Third, the H\&OP group only included female patients with gynecological tumors. This is because only a small number of patients had a benign ulcer that required stomach resection and omental preservation, and there were no eligible male patients. Fourth, because of some objective reasons, such as the need for pathological examination of omental tissue, we failed to record each omental fat volume or weight. Further clinical trials need to be designed to address these issues. Finally, whether these results will pertain to other ethnic groups or a larger number of patients is unknown.

In conclusion, resection of omental adipose tissue represents an effective strategy for reducing SBP and DBP at 8 months in patients with hypertension, even in the non-obese hypertensive population. However, this procedure has a negative effect on the normotensive population. The durability of our findings remains uncertain and the long-term effects of our procedure on metabolism are still unknown. Therefore, further study with a long period of follow-up is required. Our mRNA microarray results suggest that abnormal inflammatory or immune responses in omental adipose tissue may be an important reason for initiation and progression of hypertension, which makes omental adipose special and more important in VF. This process has no biological relationship with regional catecholamine concentrations. Taken together, our findings suggest that white adipose of the omentum may be a new target for abnormal high blood pressure, and it could provide new opportunities for future prevention, control, and even a cure for hypertension. Such effects have the potential to reduce cardiovascular events, although further clinical trials are required to determine this possibility.

\section{Statement of Ethics}

The trial was approved by the Ethics Committee of the First Affiliated Hospital of Nanjing Medical University. Written informed consent was obtained from all participants or their legal proxies.

\section{Acknowledgments}

The authors thank the Gynecological Tumor Department of Nanjing Maternity and Child Health Hospital for their assistance with this study.

\section{Author Contributions}

All authors made a significant contribution to the work reported, whether that is in the conception, study design, 
execution, acquisition of data, analysis and interpretation, or in all these areas; took part in drafting, revising or critically reviewing the article; gave final approval of the version to be published; have agreed on the journal to which the article has been submitted; and agree to be accountable for all aspects of the work. Li Jiang, Wei Sun, Mi Zhang contributed equally to this article as cofirst authors.

\section{Funding}

This work was financially supported by projects of the National Scientific Foundation of China (NSFC, grant nos. 81627802 and 81570247), the Six Talent Peaks project in Jiangsu Province (no. 2015-WSN-29), and the Priority Academic Program Development of Jiangsu Higher Education Institutions (PAPD).

\section{Disclosure}

The authors report no conflicts of interest in this work.

The abstract of this paper was presented at the European Heart Congress \& Traditional Medicine Congress as a poster presentation with interim findings. The poster's abstract was published in "Poster Abstracts" in J Cardiovascular Disease and Diagnosis Journal, 2019. The link to the abstract is https://www.hilarispublisher. com/proceedings/omental-adipose-removal-decreases-high -blood-pressure-in-hypertensive-patients-independent-ofbody-mass-index-34450.html.

\section{References}

1. Fields LE, Burt VL, Cutler JA, Hughes J, Roccella EJ, Sorlie P. The burden of adult hypertension in the United States 1999 to 2000: a rising tide. Hypertension. 2004;44(4):398-404. doi:10.1161/01. HYP.0000142248.54761.56

2. Egan BM, Jiexiang L, Hutchison FN, Ferdinand KC. Hypertension in the United States 1999-2012: progress toward healthy people 2020 goals. Circulation. 2014;130(19):1692-1699. doi:10.1161/CIRCULA TIONAHA.114.010676

3. Murray CJL; US Burden of Disease Collaborators. The state of US Health, 1990-2010: burden of diseases, injuries, and risk factors. JAMA. 2013;310(6):591-608. doi:10.1001/ jama.2013.13805

4. Lavie CJ, Alpert MA, Arena R, Mehra MR, Milani RV, Ventura HO. Impact of obesity and the obesity paradox on prevalence and prognosis in heart failure. JACC Heart Fail. 2013;1(2):93-102. doi:10.1016/j. jchf.2013.01.006

5. Borlaug BA, Reddy YN. Getting at the heart of central obesity and the metabolic syndrome: Borlaug and Reddy: metabolic heart disease. Circ Cardiovasc Imaging. 2016;9(6):pii: e005110. doi:10.1161/ CIRCIMAGING.116.005110

6. Clark JM, Brancati FL, Diehl AM. The prevalence and etiology of elevated aminotransferase levels in the United States. Am J Gastroenterol. 2003;98 (5):960-967. doi:10.1111/j.1572-0241.2003.07486.x
7. Chandra A, Neeland IJ, Berry JD, et al. The relationship of body mass and fat distribution with incident hypertension: observations from the Dallas Heart Study. J Am Coll Cardiol. 2014;64 (10):997-1002. doi:10.1016/j.jacc.2014.05.057

8. Ibrahim MM. Subcutaneous and visceral adipose tissue: structural and functional differences. Obes Rev. 2010;11(1):11-18. doi:10.1111/ j.1467-789X.2009.00623.x

9. Xia L, Hua J, Dray X, et al. Endoscopic visceral fat removal as therapy for obesity and metabolic syndrome: a sham-controlled pilot study (with video). Gastrointest Endosc. 2011;74(3):637-644. doi:10.1016/j.gie.2011.07.006

10. Schiavon CA, Bersch-Ferreira AC, Santucci EV, et al. Effects of Bariatric surgery in obese patients with hypertension: the GATEWAY randomized trial (Gastric bypass to treat obese patients with steady hypertension). Circulation. 2018;137(11):1132-1142. doi:10.1161/CIRCULATIONAHA.117.032130

11. Mancia G, Fagard R, Narkiewicz K, et al. 2013 ESH/ESC practice guidelines for the management of arterial hypertension. Blood Press. 2014;23(1):3-16. doi:10.3109/08037051.2014.868629

12. Tchernof A. Visceral adipocytes and the metabolic syndrome. Nutr Rev. 2007;J65(6 Pt 2):S24-9. doi:10.1301/nr.2007.jun.S24-S29

13. Donahue RP, Abbott RD. Central obesity and coronary heart disease in men. Lancet. 1987;2(8569):1215. doi:10.1016/S0140-6736(87) 91357-2

14. Ducimetiere P, Richard J, Cambien F. The pattern of subcutaneous fat distribution in middle-aged men and the risk of coronary heart disease: the Paris prospective study. Int J Obes. 1986;10(3):229-240.

15. Platell C, Cooper D, Papadimitriou JM, Hall JC. The omentum. World J Gastroenterol. 2000;6(2):169-176.

16. Conroy MJ, Galvin KC, Kavanagh ME, et al. CCR1 antagonism attenuates $\mathrm{T}$ cell trafficking to omentum and liver in obesity-associated cancer. Immunol Cell Biol. 2016;94(6):531-537. doi:10.1038/icb.2016.26

17. Wolf D, Jehle F, Michel NA, et al. Coinhibitory suppression of T cell activation by CD40 protects against obesity and adipose tissue inflammation in mice. Circulation. 2014;129(23):2414-2425. doi:10.1161/CIRCULATIONAHA.113.008055

18. Lysaght J, Allott EH, Donohoe CL, Howard JM, Pidgeon GP, Reynolds JV. T lymphocyte activation in visceral adipose tissue of patients with oesophageal adenocarcinoma. Br J Surg. 2011;98 (7):964-974. doi:10.1002/bjs.7498

19. Xiong XQ, Chen WW, Han Y, et al. Enhanced adipose afferent reflex contributes to sympathetic activation in diet-induced obesity hypertension. Hypertension. 2012;60(5):1280-1286. doi:10.1161/ HYPERTENSIONAHA.112.198002

20. Xiong XQ, Chen WW, Zhu GQ. Adipose afferent reflex: sympathetic activation and obesity hypertension. Acta Physiol. 2014;210 (3):468-478. doi:10.1111/apha.12182

21. Hardy OT, Perugini RA, Nicoloro SM, et al. Body mass index-independent inflammation in omental adipose tissue associated with insulin resistance in morbid obesity. Surg Obes Relat Dis. 2011;7(1):60-67. doi:10.1016/j.soard.2010.05.013

22. Rosenquist KJ, Pedley A, Massaro JM, et al. Visceral and subcutaneous fat quality is associated with cardiometabolic risk. JACC Cardiovasc Imaging. 2013;6(7):762-771. doi:10.1016/j.jcmg.2012.11.021

23. de Lemos JA, Neeland IJ. Separating the VAT from the FAT new insights into the cardiometabolic risks of obesity. JACC Cardiovasc Imaging. 2014;7(12):1236-1238. doi:10.1016/j.jcmg.2014.08.007

24. Berings M, Wehlou C, Verrijken A, et al. Glucose intolerance and the amount of visceral adipose tissue contribute to an increase in circulating triglyceride concentrations in Caucasian obese females. PLoS One. 2012;7(9):e45145. doi:10.1371/journal.pone.0045145

25. Foster MT, Shi H, Seeley RJ, Woods SC. Removal of intra-abdominal visceral adipose tissue improves glucose tolerance in rats: role of hepatic triglyceride storage. Physiol Behav. 2011;104 (5):845-854. doi:10.1016/j.physbeh.2011.04.064 


\section{Publish your work in this journal}

Diabetes, Metabolic Syndrome and Obesity: Targets and Therapy is an international, peer-reviewed open-access journal committed to the rapid publication of the latest laboratory and clinical findings in the fields of diabetes, metabolic syndrome and obesity research. Original research, review, case reports, hypothesis formation, expert opinion and commentaries are all considered for publication. The manuscript management system is completely online and includes a very quick and fair peer-review system, which is all easy to use. Visit http://www.dovepress.com/testimonials.php to read real quotes from published authors.

Submit your manuscript here: https://www.dovepress.com/diabetes-metabolic-syndrome-and-obesity-targets-and-therapy-journal 